\title{
Estudio de las interacciones escolares de estudiantes de profesiones del área de la salud
}

Marisela Rocío Soria Trujano ${ }^{1}$ y Edy Ávila Ramos ${ }^{1}$

\section{Introducción}

Son múltiples los factores que intervienen en el rendimiento académico de los jóvenes universitarios. No solamente son importantes las capacidades de los estudiantes para que logren una buena ejecución académica, también hay que tomar en cuenta las condiciones escolares: metodología docente, horarios, plan de estudios, dificultad de las materias, formación del profesorado y las relaciones entre alumnos y entre éstos y sus profesores (Garbanzo, 2007). Hay estudios que arrojan datos que indican que, además, la sobrecarga de trabajo, las evaluaciones y el tiempo limitado para realizar las tareas escolares son los estímulos estresores más frecuentemente reportados por los estudiantes universitarios (Fernández y Polo, 2011; García, Pérez, Pérez y Natividad, 2012; Valenzuela y Fraijo, 2011). Cabe señalar que muchas veces las actividades académicas pueden convertirse en un factor importante que afecta el bienestar físico de los

1 Facutad de Estudios Superiores Iztacala, unAm. Psicología. 
estudiantes, presentándose casos de dermatitis, gastritis, fatiga crónica (Mendoza et al., 2010); ansiedad y depresión (Balanza, Morales y Guerrero, 2009); baja motivación, sensación de fracaso (Casuso, 2011). En este panorama, adquieren relevancia los profesores y compañeros de escuela, puesto que pueden ser mediadores en cuanto a la presencia de ansiedad en los estudiantes (Hernández, Coronado, Araujo y Cerezo, 2008). Las redes sociales que establecen los jóvenes universitarios pueden servir como protectores para afrontar situaciones estresantes (Preciado y Vázquez, 2010), de ahí la importancia de las relaciones que los alumnos establezcan con sus compañeros y profesores. En cuanto a las interacciones entre los jóvenes, se puede decir que muchos de ellos se apoyan mutuamente para afrontar situaciones escolares estresantes, por ejemplo, para la realización de tareas académicas, para estudiar, para preparar exposiciones, etcétera. Al respecto, se puede retomar la siguiente cita: "El apoyo social es la ayuda accesible a un sujeto a través de los lazos sociales de otros sujetos, grupos y comunidad global" (Landero y González, 2002: 282). Feldman et al. (2008) proporcionan datos que apuntan al hecho de que a mayor nivel de estrés académico, los estudiantes perciben menor apoyo social. Ahora bien, en el ámbito escolar, el apoyo social no solamente se recibe de los compañeros de clase y amigos, también los profesores pueden convertirse en importantes fuentes de ayuda para los estudiantes, de ahí que las buenas relaciones entre este binomio son necesarias, debido a que los docentes en ocasiones inducen estrés a sus alumnos. Existen datos de investigación que señalan que para los universitarios es significativa la forma en la que los profesores cumplen con la función de enseñanza (pedagogía, competencias), así como el trato de éstos hacia los estudiantes, y que todo ello repercute en el rendimiento académico, observándose que la percepción de los jóvenes acerca de sus profesores es el predictor más relevante asociado al alto rendimiento (Vega y Capa, 2009). Así, surgió el interés por realizar el presente estudio cuyo objetivo fue evaluar las relaciones entre estudiantes de profesiones del área de la salud y entre éstos y sus profesores, así como la percepción de los jóvenes acerca de las condiciones 
académicas (capacidades de enseñanza del profesorado y formación que reciben los alumnos), analizando las posibles diferencias entre género y profesión.

\section{Método}

Participantes

Se evaluaron 113 estudiantes universitarios de la Facultad de Estudios Superiores Iztacala de la UNAM. Una muestra estuvo conformada por 62 alumnos de la carrera de Odontología y otra por 51 alumnos de la carrera de Biología. En cuanto a los estudiantes de Odontología, 17 eran hombres y 45 mujeres, con un rango de edad de 19 a 27 años, con una media de 21. De los participantes de Biología, 17 fueron hombres y 34 mujeres, con un rango de edad de 20 a 30 años, con una media de 22. Cursaban el sexto o el octavo semestre y realizaban servicio comunitario atendiendo pacientes o llevando a cabo prácticas de campo, además de que cursaban asignaturas de contenido teórico. Residían en la Ciudad de México o área metropolitana y se les contactó por vía de algunos de sus profesores, siendo la muestra no probabilística de tipo intencional.

Instrumento

Se diseñó una escala tipo Likert de 35 ítems y 5 códigos de respuesta: muy frecuentemente, frecuentemente, pocas veces, casi nunca y nunca. El Alpha de Cronbach fue de .788.

\section{Procedimiento}

Se obtuvo el consentimiento informado de los participantes, respetando su anonimato, haciendo de su conocimiento el uso de la información obtenida para presentarla en eventos científicos y/o para publicar. Se evaluó a los estudiantes de manera grupal en sus salones de clase. 


\section{Resultados}

Se aplicó la prueba t de Student para muestras independientes con el fin de establecer diferencias entre géneros y profesiones con respecto a las interacciones de interés. Los datos para las relaciones escolares y el género indicaron que no se encontraron diferencias significativas entre hombres y mujeres: $\mathrm{t} 111=-1.086$ $p=>.05$. Ambas muestras alcanzaron un nivel moderado de problemas en interacciones con sus compañeros y profesores (87.3\% de mujeres y $97.1 \%$ de hombres). Al analizarse los datos para cada ítem de la escala, se obtuvo diferencia significativa para el correspondiente a "Tengo problemas con algún(os) profesor(es)": $\mathrm{t} 111=2.298 \mathrm{p}<.05$, siendo las mujeres las que presentaron más dificultades. La información con respecto a los demás ítems señaló que la mayoría de los y las estudiantes frecuentemente cumplen con todas sus tareas académicas a pesar de que tienen sobrecarga de trabajo, también se relacionan con sus compañeros. No obstante, menos de la mitad de los participantes de ambos géneros reciben apoyo de sus compañeros para la realización de sus deberes escolares. Ambos géneros reportaron llegar tarde a clases frecuentemente, y tener problemas con compañeros y con algún(os) profesor(es), aunque como ya se dijo anteriormente, las mujeres manifestaron más problemas con el profesorado. La mayoría indicó satisfacción con la formación que está recibiendo, pero fueron más las mujeres quienes se sienten satisfechas con la capacidad de enseñanza de sus profesores. Algunos(as) dijeron sentirse poco motivados en el ámbito académico. Por otro lado, las comparaciones por profesión mostraron que no se encontró diferencia significativa en las relaciones escolares: $\mathrm{t} 111=.721 \mathrm{p}>.05$. Los porcentajes señalaron que los estudiantes de ambas carreras, en su mayoría, reportaron problemas en el ámbito académico (88.7 de los estudiantes de Odontología y $92.2 \%$ de los de Biología). El análisis para cada ítem de la escala arrojó datos que señalan que en ninguno se obtuvo diferencia significativa. Los porcentajes dejan ver que gran parte de los participantes de ambas profesiones cumplen con sus tareas académicas y algunas veces llegan 
tarde a clases, siendo que al relacionarse con sus compañeros surgen algunos conflictos y en ocasiones también con el profesorado. Muchos jóvenes señalaron tener exceso de trabajo académico frecuentemente, y sólo la mitad de los participantes de cada profesión recibe apoyo de sus compañeros. Fue notorio que una tercera parte de los alumnos de Odontología se sentían poco motivados en el ámbito académico, identificándose menos estudiantes así en la muestra de Biología. Algunos sujetos de ambas profesiones no se sentían satisfechos con la formación que estaban recibiendo y con las capacidades de enseñanza del profesorado.

\section{Conclusiones}

En el presente estudio se pudo observar que la mayoría de los participantes de ambos géneros y profesiones reportó tener problemas en el ámbito escolar: sobrecarga de trabajo, poca interacción y/o conflictos con compañeros y/o profesores y poco apoyo de otros alumnos. Se evidenciaron casos de insatisfacción con su formación como odontólogos o biólogos y con el nivel de conocimientos y capacidad de enseñanza del profesorado. Las relaciones que establecen los y las estudiantes pueden significar un factor importante para su desempeño académico. El apoyo entre alumnos y entre éstos y el profesorado es importante para que los jóvenes afronten situaciones escolares estresantes a las que se someten regularmente. En cuanto a la relación profesorado-alumnado, puede decirse que no sólo es indispensable la capacitación pedagógica de los docentes para favorecer un buen desempeño académico de los estudiantes, también adquiere importancia la relación entre ambas partes del binomio académico. Un profesor no sólo debe contar con los conocimientos requeridos y con la capacidad de transmitirlos, debe también mostrar una actitud positiva hacia el alumnado, estableciendo una relación basada en la buena comunicación. Los datos del presente estudio sugieren más investigación al respecto, sobre todo en relación con el rendimiento académico, 
puesto que los estudiantes del área de la salud ejercerán su profesión teniendo bajo su responsabilidad el bienestar físico de muchos pacientes. El género femenino manifestó más problemas con el profesorado, por lo cual se propone investigación en cuanto a discriminación de género y acoso en el ambiente escolar universitario.

\section{Referencias}

Balanza, S., Morales, I. y Guerrero, J. (2009). Prevalencia de ansiedad y depresión en una población de estudiantes universitarios: factores académicos y sociofamiliares asociados. Clínica y Salud, 20(2), 177-187.

Casuso, M.J. (2011). Estudio del estrés, engagement y rendimiento académico en estudiantes universitarios. Tesis doctoral. Universidad de Málaga, España. Facultad de Enfermería, Fisioterapia, Podología y Terapia Ocupacional. Recuperado de: http://scielo.sld.cu/scielo.phpscript=sci_ arttex8pid=S1727-812020100007\&Ing=es\&nrm1SO>.

Feldman, L., Goncalves, L., Chacón-Puignau, G., Zaragoza, J., Bagés, N. y de Pablo, J. (2008). Relaciones entre estrés académico, apoyo social, salud mental y rendimiento académico en estudiantes universitarios venezolanos. Univ Psychol, 7(3), 739-751.

Fernández, C. y Polo, M.T. (2011). Afrontamiento, estrés y bienestar psicológico en estudiantes de Educación Social de nuevo ingreso. EduPsykhé Revista de Psicología y Educación, 10(2), 177-192.

Garbanzo, G.M. (2007). Factores asociados al rendimiento académico en estudiantes universitarios, una reflexión de la calidad de la educación superior pública. Revista Educación, 31(1), 43-63.

García, R., Pérez, F., Pérez, J. y Natividad, L. (2012). Evaluación del estrés académico en estudiantes de nueva incorporación a la universidad. Revista Latinoamericana de Psicología, 44(2), 143-154. 
Hernández-Pozo, R., Coronado, O, Araujo, V. y Cerezo, S. (2008). Desempeño académico de universitarios en relación con ansiedad escolar y autoevaluación. Acta Colombiana de Psicología, 11(1), 13-23.

Landero, R. y González, M. (2002). Determinantes psicosociales del estrés en amas de casa. Psicología y Salud, 12(2), 279-287.

Mendoza, L., Cabrera, E.M., González, D., Martínez, R., Pérez, E. y Saucedo, R. (2010). Factores que ocasionan estrés en estudiantes. Revista de Enfermería, 4(3), 35-45.

Preciado, M. y Vázquez, J. (2010). Perfil de estrés y síndrome de Burnout en estudiantes mexicanos de Odontología de una universidad pública. Chil Neuro-Psiquiat, 48(1), 11-19.

Valenzuela, L. y Fraijo, B. (2011). Integración y estrés en estudiantes universitarios. Ponencia en el XI Congreso Nacional de Investigación Educativa, Universidad Pedagógica Nacional. Recuperado de: http://www.comie.org.mx/congreso/memoriaelectronica/v11/docs/area_16/2151.pdf.

Vega, M.L. y Capa, W. (2009). Influencia del autoconcepto, las estrategias de aprendizaje y la percepción acerca del docente en el rendimiento académico de los alumnos de Psicología, año 2007. Revista de Investigación Universitaria, 1(1), 105-114. 
